\title{
Dilemas del profesorado en la inclusión educativa de estudiantes universitarios con discapacidad
}

\author{
Professors' dilemmas in the inclusion of university students with disabilities \\ Paulina Iturbide Fernández \\ Judith Pérez-Castro
}

\begin{abstract}
RESUMEN
En este artículo analizamos los dilemas que surgen en la inclusión de las personas con discapacidad en la educación superior, a partir de dos formulaciones: la identificación y la ubicación. La investigación consistió en un estudio de caso intrínseco. Los resultados muestran situaciones dilemáticas en cuatro aspectos: la notificación previa sobre la presencia de un estudiante con discapacidad en el aula, la importancia del diagnóstico para la realización de los ajustes pedagógicos, la factibilidad de que estos alumnos cursen la educación terciaria y el tipo de carrera que deben estudiar. En los resultados también se destaca el lugar que ocupan la formación y disposición de los profesores, así como las condiciones institucionales en la atención a los dilemas.
\end{abstract}

Palabras clave: dilemas, inclusión educativa, educación superior, discapacidad.

\begin{abstract}
In this paper, we analyze the arising dilemmas in the inclusion of people with disabilities in higher education, based on two formulations: identification and location. This research consisted of an intrinsic case study. The results show dilemmatic situations in four aspects: the prior notification about the presence of a student with disabilities in the classroom, the importance of the diagnosis for the realization of pedagogical accomodation, the feasibility of these students attending tertiary education, and the type of academic program they can study. The results also highlighted the role of teachers' profile and attitude, as well as the importance of institutional conditions when dealing with dilemmas.
\end{abstract}

Keywords: dilemmas, educational inclusion, higher education, disability. 


\section{INTRODUCCIÓN}

El presente artículo es producto de una investigación cuyo objetivo fue analizar las contradicciones que surgen en el proceso de inclusión educativa de las personas con discapacidad en el nivel superior. Particularmente, lo que aquí presentamos se centra en los dilemas de identificación y de ubicación, señalados por un grupo de profesores universitarios a partir de su experiencia con estos estudiantes.

El artículo está organizado en cuatro secciones. En la primera presentamos algunos argumentos que, desde la perspectiva del dilema de la diferencia, se han elaborado en torno a la inclusión educativa; en especial retomamos la distinción que hace Brahm Norwich entre los dilemas de identificación, de ubicación y de currículum. En la segunda profundizamos en los matices que el dilema de la diferencia toma en el contexto de la educación superior, a partir de factores como el mérito, su carácter no obligatorio y la autonomía de los sujetos. La tercera sección incluye las consideraciones metodológicas que sirvieron de base para la investigación, la cual consistió en un estudio de caso intrínseco. En la cuarta exponemos los resultados que, como ya dijimos, están enfocados a los dilemas de identificación y de ubicación. Por último presentamos algunas consideraciones generales en torno a este tema.

\section{Diferencia y discapacidad: complejidades del dilema}

Los dilemas éticos son situaciones que pueden presentarse en los distintos ámbitos de la vida, ya sea individual, profesional o laboral, y en donde las personas tienen que tomar una decisión que les resulta incómoda porque conlleva una contraposición en términos de principios, valores o lealtades (Dolgoff, Harrington y Loewenberg, 2012). De acuerdo con Banks y Williams (2005), los dilemas se diferencian de otras situaciones problemáticas porque nos hacen elegir entre cursos de acción que tienen un peso similar en las consecuencias que acarrean, tanto positivas como negativas, $y$ por lo tanto generan incertidumbre.

Paulina Iturbide Fernández. Universidad Popular Autónoma del Estado de Puebla, México. Es doctora en Educación y maestra en Tecnología Educativa por la UPAEP. Directora Académica de las licenciaturas en Psicopedagogía y Pedagogía e Innovación Educativa. Participa en dos cuerpos académicos, uno sobre ética y autorregulación en los procesos educativos y otro sobre profesionalización docente. Es miembro de la Red Nacional de Educación y Valores (ReDuvaL) y de la Red Internacional de Investigadores y Participantes sobre Integración-Inclusión Educativa (RIIE). Correo electrónico: paulina.iturbide@upaep. mx. ID: https://orcid.org/0000-0003-3514-2730.

Judith Pérez Castro (autor de correspondencia). Investigador titular en el Instituto de Investigaciones sobre la Universidad y la Educación de la Universidad Nacional Autónoma de México. Es doctora en Ciencia Social con especialidad en Sociología, por El Colegio de México. Miembro del Sistema Nacional de Investigadores, nivel II. Entre sus publicaciones recientes están "Acceso a la educación y factores de vulnerabilidad en las personas con discapacidad", publicado en Voces de la Educación (2020), y el libro Discapacidad, inclusión social y educación (coord., 2019). Es miembro del Consejo Mexicano de Investigación Educativa y de la Red Nacional de Investigadores en Educación y Valores. Correo electrónico: pkjudith33@yahoo.com.mx. ID: https:// orcid.org/0000-0002-5176-0531. 
Entre los distintos factores intervinientes están las concepciones y creencias de los sujetos, los valores del contexto que rodea al dilema y su relación con la ideología dominante, los recursos materiales o simbólicos disponibles, así como la posición social de los individuos y su relación con los demás (Minow, 1990; Artiles, 1998). Un problema no se convierte por sí mismo en un dilema, sino que son los sujetos quienes lo construyen como tal, bajo determinadas circunstancias (Banks y Williams, 2005).

Particularmente sobre el dilema de la diferencia, Minow (1990) plantea que este se origina cuando se reconocen las diferencias de las personas y se les trata de manera distinta, lo que eventualmente termina por reforzar aquello que las distingue de los demás, las etiqueta y reduce sus oportunidades; sin embargo, cuando se les trata igual que al resto se corre el riesgo de no atender sus necesidades, lo que nuevamente coarta sus oportunidades. Es decir, en el centro del dilema de la diferencia está el estigma que surge al ignorar o prestar demasiada atención a un individuo o colectivo, ya que sus diferencias son interpretadas como un desvío de la norma (Minow, 1990).

El estigma es resultado del proceso disruptivo entre la identidad real y la identidad social de una persona (Goffman, 1970). Involucra situaciones en las que el individuo, clasificado como "diferente", es tratado como si fuera menos que un ser humano completo (Ainlay, Becker y Coleman, 1986). En las personas con discapacidad, el estigma del que usualmente son objeto corresponde a las categorías que Goffman (1970) denominó como las abominaciones del cuerpo, por ejemplo, en la discapacidad motriz y los defectos de carácter, como en la discapacidad psicosocial.

Como hemos dicho, los dilemas están presentes en todas las esferas de la vida. Particularmente, en la inclusión educativa, el dilema de la diferencia se refiere a:

La necesidad de dar respuestas educativas de calidad a todos los alumnos y garantizar así el derecho a la educación en entornos comunes y, al mismo tiempo, proporcionar respuestas acordes a las necesidades individuales sin renunciar a los apoyos específicos que los estudiantes pueden requerir [San Martín, 2011, p. 173].

Pero dar respuesta a la diversidad del estudiantado y mantener metas educativas comunes es una situación dilemática que puede pasar desapercibida. El docente, como agente moral, establece un debate consigo mismo para preguntarse sobre los factores y oportunidades que le pueden ayudar a asumir el reto de que todos los alumnos aprendan en igualdad de condiciones y al mismo tiempo se sientan valorados en sus diferencias (Etxeberria, 2005). La situación se complejiza porque la mayoría de los docentes tiene un margen de acción bastante acotado, debido a las exigencias institucionales, los tiempos para cumplir con los contenidos curriculares, la escasez de recursos y los múltiples trámites administrativos que les toca resolver. Además este proceso deliberativo no siempre es explícito; muchos profesores tienen que lidiar solos con sus tensiones, ya sea porque no cuentan con espacios para la discusión colegiada, porque sienten temor de lo que puedan pensar sus compañeros o simplemente porque rehúyen la discusión de manera inconsciente. 
De este modo, en la inclusión de las personas con discapacidad, la diferencia puede presentar tres formulaciones (Norwich, 2008a): 1) el dilema de la identificación, que se pregunta si se debe identificar o no a los estudiantes con dificultades y discapacidades y, de ser así, cuál sería la manera más adecuada para hacerlo, individual o colectivamente; 2) el dilema del currículum, que plantea la disyuntiva de brindar a estos alumnos los mismos contenidos de aprendizaje o enseñarles cosas diferentes que al resto de sus compañeros, y 3) el dilema de ubicación, que apunta a la pertinencia de incluir a los educandos con dificultades y discapacidades en las clases regulares o atenderlos por separado.

Estos dilemas fueron elaborados en el contexto de la educación básica. De hecho, en sus primeros trabajos Norwich (1993) propuso un cuarto dilema, referido a la relación entre los padres de familia y el profesional. Con el tiempo el autor centró su trabajo solo en los tres primeros, con el propósito de utilizarlos como marco de referencia para analizar la inclusión, la educación y otras áreas de la política social (Norwich, 2008a).

Desde otra perspectiva, Dyson y Millward (2000) señalan que los dilemas son prácticamente inseparables de los sistemas educativos, porque en ellos hay una contradicción fundamental. Fueron hechos para ofrecer educación a todos los estudiantes de un determinado territorio nacional, para lograrlo se han definido condiciones similares de enseñanza, una pedagogía compartida y un perfil común para todos los educandos. El problema es que los fines de la educación solo pueden lograrse actuando a nivel de cada sujeto, los cuales tienen diferencias importantes entre sí. De este modo, aunque pueden haber distintos tipos de dilemas, "todos surgen de la contradicción fundamental de un sistema educativo que se basa al mismo tiempo en lo que los estudiantes tienen -o se espera que tengan- en común y en las diferencias entre cada individuo" (Dyson y Millward, 2000, pp. 161-162, traducción propia).

Como cualquier otro dilema, el que se deriva de las diferencias y sus posibles formulaciones no se resuelve fácilmente; incluso cabría preguntarse si en algún momento podría llegarse a una solución, en especial de cara a la presión en la que se encuentran las instituciones educativas, que cada vez tienen que hacer más con menos. No obstante, reconocer su existencia es un paso importante en el mejoramiento de las condiciones educativas de todos los estudiantes, principalmente de los más vulnerables.

\section{E1 dilema de la diferencia en la educación superior}

Como hemos dicho, el dilema de la diferencia ha sido abordado fundamentalmente en la educación básica y la educación especial (Artiles, 1998, 2003; Dyson y Millward, 2000; Norwich, 2008a, 2008b, 2013; Echeita, 2010), sin embargo consideramos necesario y posible extender sus posibilidades analíticas al nivel superior, en donde ha habido un crecimiento lento pero constante de estudiantes con discapacidad, especialmente en las dos últimas décadas. 
Si bien los dilemas pueden presentarse en cualquier nivel educativo, en la educación básica hay algunos factores que la hacen distinta, como la relación que se establece entre la escuela y la familia, las formas de acompañamiento para atender las necesidades de los estudiantes, el tipo de barreras que se enfrentan y las estrategias para superarlas, así como el lugar que ocupan los diferentes actores educativos, maestros, padres de familia, alumnos, directivos y otros profesionales de apoyo (Norwich, 2008a).

La educación superior, por su parte, tiene una serie de rasgos que la hacen un tanto resistente a los planteamientos de la inclusión. Para los fines de este artículo nos interesa destacar tres: su carácter no obligatorio, la importancia otorgada al mérito y el peso en la autonomía de los sujetos. Con respecto al primero, Brunner (2002) plantea que a lo largo de la historia las civilizaciones han desarrollado diversas estrategias para formar a sus élites, ya fuesen sacerdotes, militares, técnicos, funcionarios o políticos. La educación superior, en particular la universidad, es solo una de estas estrategias, pero eventualmente se convirtió en el eje de la vida intelectual en casi todas las sociedades.

Durante mucho tiempo, la universidad tuvo una impronta bastante elitista, que empezó a cambiar a partir de los años 60 del siglo XX con la explosión de la matrícula. Este proceso que se dio en varios países, incluidos los de América Latina, amplió el acceso a sectores que anteriormente estaban excluidos, como las mujeres y los hijos de trabajadores (Brunner y Flisfisch, 2014; Rodríguez, 1999). Esto también permitió impulsar reformas para democratizar la universidad, las cuales, sin embargo, no lograron abarcar a todos los colectivos (Juarros, 2006), entre ellos las personas con discapacidad, que continuaron excluidas de los estudios profesionales.

No obstante, a finales del siglo XX con el fortalecimiento de la inclusión educativa y los movimientos a favor de los derechos de la población con discapacidad (Moola, 2015; UNESCO, 2009), paulatinamente estas comenzaron a formar parte de la demanda educativa, de manera que ahora las instituciones tienen que "estar disponibles para todos los estudiantes y, en especial, para aquellos en situación de vulnerabilidad" (Gairín y Suárez, 2016, p. 2).

De este modo podemos dar cuenta de un primer aspecto del dilema de la diferencia. Por un lado la obligación que asumen el Estado y sus instituciones de garantizar las oportunidades de este colectivo para ingresar y concluir exitosamente la educación superior, y por el otro la falta de condiciones para incluirlos de manera plena en los establecimientos educativos. Como consecuencia se generan diversas formas de inclusión excluyente (Terigi, 2009), por ejemplo, se les permite ingresar pero les es muy difícil permanecer debido a las barreras que enfrentan, o bien permanecen pero obtienen calificaciones bajas, adeudan cursos o tienen trayectorias discontinuas.

El segundo rasgo que queremos señalar es la importancia otorgada al mérito. Para Dubet (2004), el mérito es un componente inseparable de todos los niveles educativos. Avanzar en el sistema escolar depende en gran medida del esfuerzo y la 
capacidad de cada individuo. En el caso de la educación superior, quienes llegan son aquellos que lograron sortear los obstáculos a lo largo del sistema de enseñanza y por lo general los que tienen mejores calificaciones (Juarros, 2006).

Las personas con discapacidad, además de los problemas comunes a todos los estudiantes, afrontan desventajas adicionales. En primer lugar, los que estudiaron en la educación especial no siempre tienen un perfil que les permita competir con sus compañeros egresados del sistema regular; en segundo, aquellos que se formaron en instituciones regulares y no recibieron los apoyos necesarios suelen tener un desempeño más bajo tanto en sus habilidades y competencias como en sus calificaciones (OECD, 2003). Todo esto se concreta en múltiples barreras, por ejemplo para atender los requisitos de ingreso, en especial para las carreras con mayor demanda o prestigio, o bien para adecuarse a los formatos y tiempos del proceso de admisión, aunque tengan los conocimientos necesarios. Al respecto podríamos preguntarnos: ¿De qué manera se puede valorar el mérito y al mismo tiempo reconocer las diferencias de los estudiantes?, ¿es justo colocar a los alumnos con discapacidad en las mismas condiciones que sus pares cuando las dificultades que enfrentan son mayores?

Una vez en la educación superior, dos de los factores más importantes para la permanencia de todos los estudiantes son las calificaciones y el proceso de adaptación a la institución, no obstante, las personas con discapacidad necesitan de ciertos apoyos, como materiales accesibles, ajustes a los contenidos de los programas, tutorías y tiempos diferenciados para la entrega de los trabajos, entre otros (Fuller, Bradley y Healey, 2004). Aquí la pregunta es: ¿Qué estrategias permitirían brindar a estas personas condiciones equitativas para su permanencia, sin caer en la simulación o el asistencialismo? Es decir, se trata de un tema de igualdad de resultados a partir de diferentes puntos de partida (Latapí, 1993).

El riesgo que se corre es que si no se ofrecen los suficientes apoyos se incrementan las posibilidades de no lograr un buen desempeño o de generar recorridos atípicos, lo que al final transmite una idea de fracaso individual que se asocia a la discapacidad. Contrariamente, si los apoyos no se distribuyen de manera clara y equitativa pueden surgir cuestionamientos respecto al desempeño de estos alumnos, en especial de cara a sus compañeros que no recibieron apoyos específicos.

El tercer aspecto que nos interesa destacar es la importancia otorgada a la autonomía de las personas. En la educación superior se espera que los individuos que ingresan sean adultos, autónomos y "normales" (Borland y James, 1999; Moola, 2015). Esta imagen se desdibuja en los estudiantes con discapacidad, porque el estigma que los coloca como débiles, enfermos o incapaces lleva a pensar que ellos no encajan en la institución. Sin embargo el hecho de que requieran apoyos y ajustes no los hace menos autónomos ni mengua su dignidad, más aún si consideramos que todos podemos necesitar ayuda en algún momento de nuestras vidas, ya sea por enfermedad, por accidente, en las mujeres por el embarazo o por alguna otra eventualidad. Pero, como 
bien advierte Nussbaum (2007), estas deficiencias suelen verse como "normales", comunes y sobre todo temporales; situaciones que los individuos pueden superar en un periodo razonable para después reincorporarse plenamente a la dinámica que sigue la mayoría, algo que no se espera de las personas con discapacidad. Sobre esta base, muy frecuentemente se invalida su capacidad y derecho para tomar parte de los diversos ámbitos de la vida social, en este caso la educación superior.

Como hemos señalado, estos son solo algunos de los múltiples factores que intervienen en el dilema de la diferencia en la educación terciaria. Sus efectos en la inclusión de las personas con discapacidad y sus posibilidades de resolución dependen, entre otras cosas, de los recursos y condiciones institucionales, de la formación y disposición de los profesores, directivos y estudiantes con y sin discapacidad, así como de la capacidad para implementar acciones concertadas entre ellos.

\section{ENFOQUE DE LA INVESTIGACIÓN Y MÉTODO}

El presente artículo es producto de una investigación desarrollada a partir de un estudio de caso intrínseco (Stake, 1999). El trabajo empírico lo realizamos en una universidad privada del estado de Puebla. De acuerdo con los datos del INEGI (2013), esta entidad concentra el $44.3 \%$ de las personas con discapacidad del país. A nivel estatal se reportan 63,575 personas con discapacidad visual, 27,584 con discapacidad auditiva, 28,926 con discapacidad intelectual y 119,109 personas cuentan con discapacidad motriz. Solo el $4.1 \%$ de las personas de este colectivo ha podido acceder a la educación superior, lo que representa una minoría y, con ello, un problema a estudiar ya que, de acuerdo con el Consejo Nacional para Prevenir la Discriminación (ConApred, 2010), el 43.3\% de personas con discapacidad del país que asiste a la universidad señala que sus derechos no son respetados.

Utilizar este método fue pertinente porque nos permitió acercarnos a la dinámica institucional que enmarca las prácticas del profesorado para la atención a la diversidad y al mismo tiempo nos ayudó a comprender la complejidad de dicho proceso a partir de las experiencias, principios y valores que están presentes en el proceso de inclusión. Para seleccionar el caso, siguiendo a Stake (1999), construimos los siguientes ejes temáticos:

1) La filosofía y los valores institucionales. Implicó la revisión de la normativa, planes de desarrollo, programas y acciones educativas, para ver la importancia que el enfoque de la inclusión educativa tiene en el discurso institucional. La universidad seleccionada para nuestro estudio se fundamenta en la filosofía humanista y en valores como la verdad, el bien, la integridad de la persona humana, la libertad, la solidaridad, la subsidiaridad y la justicia, entre otros. Específicamente, respecto a las personas con discapacidad, lo anterior se ha concretado en acciones como la realización de ajustes en el proceso de ad- 
misión; la firma de convenios con instancias gubernamentales y asociaciones de la sociedad civil que trabajan con grupos vulnerables; la adaptación de las instalaciones universitarias y la implementación de materiales accesibles en algunas de las carreras. No obstante, a la fecha la universidad no cuenta con una política integral dirigida a la población estudiantil con discapacidad, el único colectivo para el que la universidad se ha desarrollado un programa de atención es el de los alumnos provenientes de comunidades indígenas y rurales.

2) La presencia de estudiantes con discapacidad. En esta dimensión queríamos ver la proporción de personas con discapacidad en la matrícula institucional y su evolución en los últimos años, pero al revisar los informes observamos que no hay estadísticas oficiales sobre estos alumnos y tampoco existe un departamento que les dé seguimiento, lo que nos llevó a recabar la información a través de los registros de cada una de las carreras. En el periodo en que se realizó la investigación la universidad tenía una matrícula de 9 mil estudiantes a nivel licenciatura, de los cuales seis tenían una discapacidad. Esto fue muy importante para nuestro estudio, ya que algunos de los valores que defiende la universidad, como la justicia y la integridad de la persona, coinciden con el enfoque de la inclusión educativa, de ahí que resulte extraño que no cuente con información sobre los alumnos con discapacidad ni con una estrategia que permita acompañarlos a lo largo de su trayectoria.

3) El lugar de los profesores en la inclusión de los estudiantes. Aquí nuestro interés era acercarnos al conocimiento que el personal docente tenía respecto a la inclusión de las personas con discapacidad, los procesos de formación tanto en el ámbito de la didáctica general como de la didáctica para la atención a la diversidad, así como su experiencia previa con estos alumnos. Encontramos que, para la universidad, el perfil de sus docentes debe atender a tres dimensiones, la personal, la profesional y la pedagógica, aunque en ninguna de ellas se considera la formación para la inclusión. A pesar de esto, en los años recientes se han promovido algunos espacios de formación, como talleres, asesorías y sesiones de acompañamiento para los profesores que tienen estudiantes con discapacidad, así como conferencias y foros para la sensibilización de la comunidad institucional.

Con base en estos elementos, consideramos que seleccionar a esta universidad nos daba posibilidades para acercarnos a los dilemas que surgen en la inclusión de los estudiantes universitarios con discapacidad, ya que, como vemos, la institución se encuentra en un momento en el que busca ampliar su cobertura hacia ciertos sectores vulnerables, pero aún no existe una política de inclusión que articule las acciones que se han desarrollado hasta ahora. 


\section{Técnica de la investigación}

Para trabajar con los sujetos de estudio utilizamos la entrevista a profundidad, la cual es una técnica holística que nos permite obtener información contextualizada y personalizada (Valles, 1999). Diseñamos un guión organizado a partir de tres dimensiones: 1) El perfil de los profesores universitarios, 2) los conflictos en la atención de estudiantes con discapacidad y 3) los dilemas docentes en el proceso de inclusión.

El instrumento se validó a través del juicio de expertos. Para ello se sometió a una revisión con dos especialistas nacionales y dos internacionales. Todos ellos tenían estudios de doctorado, tres eran especialistas en inclusión educativa y discapacidad y uno en ética profesional. Los dos expertos mexicanos pertenecían al Sistema Nacional de Investigadores y los internacionales tenían una larga trayectoria de proyectos de investigación y producción académica en las temáticas señaladas, además de ser autores referenciados en el campo de la inclusión.

Con base en las observaciones de los expertos se hicieron los ajustes y después el guión se piloteó con una profesora de la universidad que había trabajado con un estudiante con discapacidad. Con las repuestas obtenidas se hicieron nuevas adecuaciones, principalmente en la redacción de las preguntas. También se incorporó un dilema ético que sirviera de referencia a los entrevistados y se precisaron algunas palabras para que fueran más adecuadas al perfil académico. Después de todo este proceso se logró la versión final.

\section{Análisis de la información}

Para esta fase de la investigación, primero se transcribieron las entrevistas, las cuales tuvieron una duración promedio de una hora 30 minutos. Como técnica de análisis decidimos utilizar el bricolaje, que consiste en "una combinación ecléctica de formas múltiples de análisis, y una interpretación de inspiración teórica de las entrevistas como modo significativo de análisis" (Kvale, 2011, p. 137). Como segundo paso realizamos un análisis de contenido de cada entrevista de manera separada, codificamos de forma manual para identificar los elementos que conformaban cada dilema y, sobre todo, para distinguirlos de asuntos o conflictos éticos que no llegaban a ser parte de ellos. Posteriormente construimos categorías siguiendo el orden de las dimensiones establecidas en el guión de entrevista. Por último hicimos un análisis transversal para identificar las similitudes y diferencias entre lo señalado por los entrevistados, así como para identificar los dilemas más importantes.

\section{Sujetos de estudio}

Para el acercamiento a los dilemas tomamos como base la perspectiva de los profesores, ya que son ellos los que tienen el reto de incluir a los estudiantes con discapacidad en la cotidianidad de las clases. La selección de los informantes se realizó 
mediante un muestreo intencional y de sujetos voluntarios; una profesora no accedió a ser entrevistada, por lo que se seleccionó a otro docente. El primer paso fue ubicar a los estudiantes con discapacidad que, para el momento de nuestra investigación, eran seis, inscritos en las carreras de Cine, Enfermería, Educación, Humanidades y Psicopedagogía. Cuatro de ellos habían realizado cambio de carrera. Al momento del estudio, un alumno se encontraba cursando el semestre 13, ya que había tenido una trayectoria irregular; otro joven estaba inscrito en 80 . semestre y el resto se ubicaban entre el 1o. y el 3er. semestres. Sus edades oscilaban entre los 18 y 22 años. Con respecto a los tipos de discapacidad, tres estudiantes tenían discapacidad visual, uno motriz, uno discapacidad auditiva y uno más psicosocial.

El segundo paso fue elegir a dos por profesores por alumno, de los cuatro o cinco que les habían impartido clases en el semestre. Los criterios de selección fueron: ser docente activo en la universidad, impartir clases a nivel licenciatura en la modalidad escolarizada y haber tenido algún estudiante con discapacidad en cuando menos una de las asignaturas impartidas durante periodo académico previo a la entrevista.

De los 12 profesores que conformaron la muestra final, seis eran hombres y seis mujeres. Respecto a la edad, tres tenían entre 30 y 39 años, cinco estaban entre 40 y 49 , cuatro entre 50 y 59 años y uno era mayor de 60. Dos contaban con una antigüedad de menos de nueve años en la institución, seis tenían entre 10 y 20 años de experiencia y tres más alrededor de 25 años, es decir, la mayoría eran docentes con una larga trayectoria en la universidad y con conocimiento de la dinámica institucional. Además los entrevistados impartían clase en carreras relacionadas con su perfil profesional, todos tenían experiencia laboral en su campo y dijeron haber tomado cursos de formación pedagógica recientemente. Por último, solo dos tenían experiencia o conocimientos previos sobre los temas de inclusión y discapacidad.

\section{Consideraciones éticas}

Para este trabajo tomamos como base la propuesta de Miles, Huberman y Saldaña (2014), para quienes la ética de la investigación debe apegarse a cuatro elementos fundamentales: 1) comunicar sus objetivos a los sujetos de estudio, 2) contar con el consentimiento informado y voluntario, 3) establecer una relación de confianza y transparencia entre el investigador y los participantes y 4) considerar la reciprocidad en el proceso investigativo.

Con base en lo anterior, buscamos establecer una relación directa con nuestros informantes, les explicamos los fines de nuestra investigación y solicitamos su consentimiento informado, el cual fue planteado y grabado al inicio de cada entrevista. Además les dejamos claro que tenían la posibilidad de abstenerse de responder las preguntas que consideraran incómodas y de solicitar más información sobre los resultados. Para guardar la confidencialidad, a cada informante le asignamos el código 
P (de profesor), un número consecutivo y las tres primeras letras de la carrera. Este mismo proceso se siguió para mantener el anonimato de los estudiantes, cuando los entrevistados se refirieron de manera directa a ellos sustituimos sus nombres por el código E, más el número correspondiente al orden en que fueron entrevistados y las tres primeras letras de la licenciatura.

\section{Resultados}

Como hemos señalado, el objetivo de este artículo es analizar los dilemas que surgen en la inclusión de las personas con discapacidad en la educación superior, partiendo del reconocimiento de que estas instituciones no fueron pensadas para responder a la diversidad, no solo en la parte física sino sobre todo en su organización académica (OECD, 2003). Como consecuencia, para los profesores incluir a este sector de la población genera una serie de tensiones, ya que intentan compaginar las necesidades de los estudiantes con los estándares institucionales.

Para presentar los resultados nos basamos en la clasificación de Norwich (2008a) y especialmente nos enfocamos en el dilema de ubicación y en el de identificación, que fueron los más señalados por nuestros sujetos de estudio.

\section{Las disyuntivas de la identificación}

Saber con anticipación sobre la presencia de un alumno con discapacidad en el aula puede ser un importante facilitador, ya que el docente tiene la oportunidad de prepararse con anticipación, hacer ajustes a la planeación didáctica, buscar materiales accesibles y diseñar estrategias inclusivas. No obstante, al mismo tiempo puede ser una desventaja por la incertidumbre que genera cuando este siente que está poco preparado, que no dispone de las estrategias pedagógicas adecuadas o incluso cuando cree que estos estudiantes le pueden causar problemas a lo largo del curso.

$\mathrm{Al}$ respecto nuestros entrevistados señalaron que tener a una persona con discapacidad en la clase les produjo desconcierto, extrañeza y miedo, porque no conocían los ajustes que podían hacer ni cuál era el camino más conveniente para establecer una relación académica con él/ella. De los doce entrevistados, siete no recibieron aviso previo por parte del director o tutor de la carrera. De este modo, la primera dificultad surgió en el momento en que el profesor entró al salón de clases y se percató de la presencia del estudiante. Una profesora de la carrera de Humanidades señaló lo siguiente:

En el caso de E1-HUM, él llegó sin que se nos avisara nada ¿no? Entonces, claro, tú tienes un alumno que notas diferente, pero no sabes qué tiene y no sabes cuáles son sus circunstancias. Entonces, si tú no tienes ese antecedente, es muy difícil que desarrolles una estrategia [...] Como docente, entender cuáles son sus intereses, cuáles son sus motivaciones, sus habilidades, sus destrezas, sus limitaciones; entonces, es difícil también no saber si acercarte a él y preguntarle, o sea, tampoco, ¿cómo lo haces, pues? [P12-HUM]. 
En esta situación el dilema se centró en la pertinencia de acercarse directamente a la persona y preguntarle sobre su condición o bien pasarlo por alto y tratarlo de la misma manera que al resto de sus compañeros. Lograr un balance entre estas dos posturas no es sencillo, porque si el profesor le pregunta corre el riesgo de resaltar su diferencia, pero si no lo hace, muy probablemente no pueda brindarle los apoyos y ajustes que requiere (Norwich, 2008a).

En el área de Enfermería, el temor de no sentirse preparado también fue señalado. Los dos profesores entrevistados coincidieron en lo complejo que para ellos fue trabajar con un alumno con discapacidad. Uno de ellos señaló:

Yo me acerqué a él porque lo vi con los aparatos. Entonces, un día lo llamé terminando la clase y le dije: “¿Te puedes quedar conmigo?”, y ya que le dije: “Oye, noto que traes aparatos”, me dijo: “Sí", le dije: “¿Me escuchas?”, me dijo: "Sí”, y le dije: "Bueno” [...] "si tú no escuchas lo que yo estoy diciendo, levanta la manita y pregúntame". Esa es otra de las cosas, con discapacidad, desde el principio, se debió haber acercado él a mí a decirme: "Oiga, maestro, tengo esta discapacidad, así y asî". Nunca lo hizo [P9-ENF].

Como vemos aquí, el dilema se vincula con el tema de quién es el responsable de notificar sobre la condición del educando, la institución o el propio estudiante. Por lo general, en el nivel superior se parte de la idea de que es este último quien debe dar cuenta de sus necesidades, porque se trata de personas adultas y autónomas (Borland y James, 1999; Moola, 2015). Cuando no hay claridad en lo que le corresponde a cada una de las partes pueden crearse conflictos en el proceso de enseñanza, así como en la relación entre los actores educativos, el alumno, el docente y la universidad.

Al respecto, Getzel (2008) señala que por lo general la autoidentificación es una situación problemática para los estudiantes con discapacidad porque, además de enfrentar el reto de cumplir con las exigencias académicas al igual que el resto de sus compañeros, tienen que lidiar con las barreras institucionales, y muchas veces con el temor derivado de las malas experiencias que tuvieron en otros niveles educativos. Ingresar a la educación superior sin la preparación necesaria y desconocer los servicios disponibles en la institución contribuye a que estos alumnos se resistan a autoidentificarse por miedo a ser etiquetados, o bien que decidan esperar hasta tener algún problema académico para hacerse visibles.

Por otro lado, en nuestra investigación también encontramos profesores que sí fueron notificados anticipadamente sobre la presencia de una persona con discapacidad. Ellos señalaron que contar con esta información les dio una idea, aunque muy general, de los retos que podían enfrentar, pero esto no estuvo exento de problemas. Para los cuatro entrevistados en esta situación el dilema se centró en las expectativas que elaboraron respecto a estos educandos, las cuales usualmente son bajas, y en el compromiso de acompañarlos en su formación profesional, asumiendo la tarea de reconocer otras capacidades que les permitieran avanzar. 
En esta formulación del dilema de identificación el impacto no se produce en el momento en que el docente llega al aula sino antes, porque están de por medio los prejuicios y etiquetas socialmente construidos sobre la discapacidad (Norwich, 2008a). Esto determina su actuación, muchas veces generando rechazo o sobreprotección hacia estos estudiantes (Etxeberria, 2005). Uno de los entrevistados comentó sobre su experiencia:

Bueno, la verdad es que sí me informaron del departamento [...] los directivos nos informaron que iba a haber una persona con una discapacidad, Asperger, y obviamente uno no sabe mucho de eso, pero sabe que tiene que ver con el... pues, si no es un caso gravísimo de innatismo, pues son personas super introvertidas, ¿no? Yo solo sabía eso [...] La verdad es que cuando me dijeron, o sea, al inicio, la primera noticia fue un impacto total porque dije: "No", o sea, ¿cómo aceptas una..., no? Cuando yo ya la conocí y le empecé a dar clases, cuando yo entré [al salón], o sea, nada de mis expectativas se cumplió [P2-HUM].

Muchas veces lo que está en juego es el poco conocimiento que los profesores tienen respecto a la discapacidad. Ante esto, uno de los profesores buscó información sobre el tema para sentirse más preparado; otros dos preguntaron directamente al estudiante sobre sus necesidades, al tiempo que contactaron al coordinador de la carrera para solicitar orientación, y el último esperó a que fuera el alumno quien solicitara los apoyos. En el caso de la carrera de Cine, uno de los profesores abundó:

Me comentó el coordinador y, de hecho, tenemos una reunión anual con los padres de los chicos, que es algo inusual [...] Creo que tenemos que prepararnos mejor. Todos nuestros contenidos y herramientas, todo debía estar, ser lo suficientemente flexible como para darles cabida. Y, bueno, tengo la impresión, realmente no estoy tan familiarizado, pero mi cabeza estaba dando vueltas pensando en lo frágil que es ir en esa dirección, nuestro modo de abordar las cosas, porque es más bien rígido y hecho para gente que tiene ciertas características que consideramos normales... [P7-CIN].

Como vemos, el docente reconoce que no basta con tener información sobre el alumno sino que es necesario estar preparado y tener competencias adecuadas para manejar esta situación. La comunicación con el estudiantado, la reflexión sobre su propio trabajo, la planificación basada en las diferencias, la colaboración entre colegas y la formación continua son elementos que pueden ayudar a comprender y responder adecuadamente a la diversidad (Amaro, Méndez y Mendoza, 2014). En nuestra investigación la mayoría de los entrevistados mostraron disposición e interés para atender a los educandos, aunque no siempre lograron entablar un diálogo, ni con sus pares académicos ni con la institución, que les permitiera compartir sus experiencias, innovar sus prácticas, aprovechar los recursos existentes o plantear sus necesidades formativas sobre el tema de la inclusión.

Los doce profesores coincidieron en que trabajar con personas con discapacidad era una tarea complicada en la que se sentían solos y para la que necesitaban orien- 
tación por parte de la universidad. Como señala Norwich (2010), muchos docentes responden a este dilema con voluntad y compromiso, los cuales, aunque son muy importantes, resultan insuficientes; se necesita que a la par las instituciones flexibilicen sus servicios, mejoren sus condiciones y cuenten con personal especializado que los apoye a lo largo del proceso.

Otra formulación del dilema de identificación está relacionada con el diagnóstico, es decir, con conocer la condición de la persona con discapacidad desde una perspectiva especializada, que sirva como punto de partida para la relación pedagógica entre el docente y el estudiante. En este caso la mitad de los entrevistados plantearon que por un lado el diagnóstico era importante porque permitía tener más elementos para incorporarlos en la planeación didáctica, mientras que por el otro admitieron que esto podía limitar la mirada únicamente en las deficiencias del alumno. Un profesor de Psicopedagogía señaló:

...si lo vemos desde el punto de vista de la discapacidad y está diagnosticado y él tiene posibilidades, digamos, a su manera, pero acomodando la clase a las posibilidades de la persona, sí es posible. O sea, yo estaría, digamos, no tendría ningún problema. Pero, ¿qué hay detrás de todo eso? Es lo que yo me pregunto con el caso de este alumno, porque ha habido, yo creo, no sé si una compasión. Yo le dije: “¿Crees tú que tengas algún otro tipo de necesidad?, ¿ya te has hecho otros diagnósticos, tus papás te han llevado, tienes terapias? [...] ¿Ya tienes los resultados, en qué te puedo ayudar?” [...] pero, pues, también él no me decía nada [P5-PSI].

Como vemos aquí, el diagnóstico se entiende como una base para la realización de los ajustes. Sin embargo, desde la mirada individualizante y rehabilitadora se corre el riesgo de patologizar, medicalizar y exacerbar las carencias de las personas (Affonso, Collares y Untoiglich, 2013), al tiempo que la responsabilidad de adecuarse a las condiciones de la clase o de la institución recae únicamente en el educando.

El problema es que el diagnóstico está vinculado con los enfoques deficitarios sobre la discapacidad (Dyson y Millward, 2000) y durante mucho tiempo se utilizó como criterio para la exclusión de estas personas; insistir en ello puede traducirse en barreras adicionales tanto para los estudiantes como para el desarrollo de prácticas inclusivas y, una vez más, reforzar los estigmas en torno a la discapacidad (Booth y Ainscow, 2002). De esta manera, el reto es hacer que la información sobre la condición del educando no sea un requisito inamovible para su ingreso a la universidad y que tampoco se convierta en otra fuente de obstáculos, sino que sirva de base para construir ambientes pedagógicos en donde todos se sientan incluidos y respetados en su dignidad.

Llama la atención que, de los doce entrevistados, solo uno no hizo énfasis en el diagnóstico, no obstante esto obedeció más bien al poco interés por estos alumnos y no a una actitud incluyente. A continuación su testimonio:

En el caso de E6-HUM y de E1-HUM no había ningún problema. Yo, mientras no me... si no me decían nada, yo me seguía a mi paso y lo agarraron. Como ellos se adaptaron yo me seguí. 
Hubiera yo actuado de otra manera si me hubieran dicho o hubiera yo notado que no podían mantener ese paso [...] Pero, pues están allí [en la clase], tienen cómo hacer las cosas [P11-HUM].

Como hemos señalado, la inclusión en la educación superior requiere tanto de la disposición y conocimiento del profesor como de las condiciones y recursos institucionales. En nuestra investigación, nueve de los entrevistados sostuvieron que la universidad era la que debía brindarles información específica sobre los alumnos con discapacidad y decirles cómo realizar las adecuaciones, ya sea a partir de acciones trabajadas con ellos previamente o bien desde algún área especializada en temas de discapacidad. Es decir, hay una tendencia a delegar la responsabilidad en el estudiante o en la institución, y en caso de tener resultados poco favorables los señalamientos se centran en la falta de apoyos o en la discapacidad del educando, en lugar de asumir su rol como copartícipes en el proceso.

\section{Las contradicciones de la ubicación}

Otro dilema identificado por Norwich (2008a) es el de la ubicación, que, referido a la educación básica, se pregunta sobre la conveniencia de que los niños y niñas estudien en la escuela regular o si es mejor enviarlos a la educación especial. En nuestra investigación identificamos dos formulaciones para este dilema: la primera tiene que ver con la disyuntiva de si las personas con discapacidad pueden y deben estar en la educación superior o si tendrían que ser canalizados a otro tipo de institución, mientras que en la segunda la pregunta es si el tipo de discapacidad debe ser o no un elemento a considerar para admitirlas en determinadas carreras. Un docente de Humanidades expresó:

O sea, somos una universidad de valores, que creemos en la persona y todas las personas por ser personas valen, ¿no? [...] O sea, no sé, desde el punto de vista humano creo que me da gusto, pero si empezamos a poner otros criterios habría que preguntarse, si, o sea, por ejemplo, los hacemos hacer la universidad, les damos un título que evidentemente no lo sacó con los mismos criterios que otros y, entonces, ¿ahora qué?, ¿le vamos a dar el trabajo?, entonces, ¿la universidad los va a contratar o quién los va a contratar?, ¿'los que los contraten van a saber que están así? [P2-HUM].

En este testimonio el dilema de ubicación se subdivide en dos vertientes, por un lado, si se acepta que las personas con discapacidad deben tener un lugar en la universidad, cuál es la responsabilidad que tendría que asumir la institución; por el otro, si concluyen la educación superior, cuáles serían sus posibilidades de ingresar al mercado laboral. Cinco de los profesores entrevistados enfatizaron este cuestionamiento, aun sabiendo que existe un riesgo de exclusión en ello. Una profesora de Humanidades comentó:

Pienso que es totalmente válido. Digo, yo trabajo el tema de la diversidad cultural y de la inclusión, entonces, para mí es totalmente válido. Pero también creo que se corre el riesgo, ¿̇no?, a veces por hacer lo políticamente correcto o por tener miedo a las demandas o por... ¿¿no?, que no 
establecemos criterios claros y me parece que son muy importantes. Es decir, claro, la universidad tiene un sentido, tiene objetivos, tiene que desarrollar ciertas habilidades en los estudiantes, entonces, también habría que ver si todo tipo de discapacidad tiene que entrar aquí [P12-HUM].

Como se observa, los docentes coinciden en que es muy difícil conciliar la atención a la diversidad con las exigencias de la educación superior. Predomina la perspectiva deficitaria de la discapacidad, según la cual las personas con esta condición de vida nunca serán plenamente capaces y, por lo tanto, su formación difícilmente cumplirá con el canon establecido para los demás estudiantes, ni tendrán las mismas oportunidades para conseguir un empleo. En nuestros entrevistados la preocupación se centra en si todos los tipos de discapacidad deberían tener cabida en la universidad y si la institución está preparada para ello, pero no en las barreras que impiden que estos educandos cursen la educación terciaria en igualdad de condiciones que sus compañeros, en las acciones que se tendrían que hacer para superarlas, ni en la manera en que los profesores pueden aportar a ello.

Otro de los aspectos mencionados por los docentes fue el riesgo de caer en el asistencialismo o paternalismo, ya que, al tener una filosofía humanista, la universidad de alguna manera puede sentirse obligada a dar un lugar a estas personas, sin que previamente haya diseñado una política integral para la inclusión. Una profesora de la licenciatura en Educación planteó lo siguiente:

Dentro de este punto, creo que necesitamos ubicar bien a quién realmente sí podemos acompañar y a quiénes, realmente, aunque queramos y podamos acompañarlos, la misma persona, no va a poder ser, ¿no? Yo no sé, yo no sé si podría hacer un estilo como un currículo excesivamente flexible. No tengo ni idea, de que a lo mejor no terminen la licenciatura, que se les dé algún diplomado o alguna cosa. $\mathrm{O}$ a lo mejor darles como opción, y no porque sea aventarlos a nuestro sistema de universidad abierta, pero darles una opción [P5-EDU].

Lo aquí señalado está en sintonía con lo expresado por la anterior docente, quien se preguntaba si la institución no estaría renunciando a su responsabilidad de brindar una buena formación por "hacer lo políticamente correcto". Sería interesante saber si este tipo de señalamientos se hacen en el caso de las personas sin discapacidad, que también pueden enfrentar múltiples obstáculos para permanecer y terminar la universidad, así como para ingresar al mercado laboral.

La segunda formulación del dilema de ubicación que enunciábamos al inicio de este apartado es sobre la relación entre la discapacidad y la carrera a elegir. En la mayoría de las instituciones, para ciertos programas hay una mayor exigencia en el perfil de ingreso de los alumnos, en términos de conocimientos y habilidades, la cual se remite a la distinción entre las profesiones liberales, altamente valoradas en el mercado, y profesiones salariales, que por lo general dependen de las instituciones públicas para poder existir (Dubet, 2004). Esto se refleja en los testimonios en algunos profesores que consideran poco realista que una persona con discapacidad sea admitida en algunas carreras. Un docente señaló: 
Probablemente, bueno, él es inteligente, no es probablemente, es inteligente, que pueda encauzar su desarrollo hacia la investigación, hacia la lectura, más a la investigación, análisis, menos práctica $[. .$.$] Entonces, esta persona que se enfrente a un paciente, en automático lo va a recha-$ zar, y entonces, ¿a qué enfrento a ese alumno? Es más, antes del paciente, el mismo personal, son discriminativos, somos muy, muy, muy discriminatorios, es la palabra, no puedo encontrar otra [...] Porque, le digo que, ¿cómo va a terminar esta asignatura y qué va a hacer? Ya terminó [enfermería], ¿después qué? Pues, porque va, va a ir un servicio social y servicio social me los acaban mucho a mis niños, ¿dónde va a hacer servicio social? [P10-ENF].

Pero esta idea no solamente está entre el profesorado, sino que es un razonamiento que incluso comparten las familias de algunos estudiantes. Una profesora señaló:

Con este chico fue completamente distinto, o sea, un día, el último día de inscripciones del semestre, llegó, tocó mi puerta con su papá y me dijo: "Maestra, me manda el coordinador porque, este, estoy inscrito en Humanidades" [...] Empecé a platicar con él, con el papá, bueno, “¿cómo fue que llegaron aquí?, ¿cómo te llamas?, ¿cuántos años tienes?” Y ahí me empecé a enterar de todo su historial, había ya estudiado o intentado estudiar en dos programas previos [...] cuando su papá me dijo que porque no había matemáticas [en esta carrera], dije: “¡No!”, casi me da el infarto, porque yo he tenido toda mi vida que luchar contra ese prejuicio de que las humanidades son super fáciles [P1-HUM].

Como vemos, las etiquetas atribuidas a las personas con discapacidad no son exclusivas de un grupo en particular. En los individuos "de a pie", incluyendo muchas veces familiares y amigos, la idea de que solo las personas "normales" deben estudiar la universidad está bastante extendida, lo anterior se manifiesta de muchas formas, desde el rechazo directo hasta la lástima bien intencionada (Moola, 2015). En los establecimientos educativos estas ideas y actitudes se reconfiguran, a veces se matizan y en otras se refuerzan, como resultado de los intereses de los diferentes actores educativos y la posición que ocupan en la estructura institucional.

Por último, de acuerdo con lo señalado por nuestros entrevistados, el dilema de ubicación es resultado del choque que se produce entre los prejuicios respecto a las personas con discapacidad, el reconocimiento del esfuerzo que han hecho para llegar a la universidad y la factibilidad de que la institución responda adecuadamente o no a sus necesidades.

\section{Consideraciones FINALES}

La inclusión educativa conmina a los sistemas e instituciones a brindar una educación en igualdad de condiciones para todos los estudiantes, y que al mismo tiempo responda a sus diferencias y necesidades. No obstante, este compromiso puede ocasionar tensiones entre los distintos actores educativos.

En este artículo nos hemos centrado en los dilemas de identificación y de ubicación señalados por un grupo de profesores universitarios. Con respecto al primero podemos destacar la importancia de construir una red de colaboración entre el 
estudiante, los docentes y la institución, ya que no basta que el profesor sepa con anticipación que tendrá a una persona con discapacidad en su clase sino que, a la par, debe recibir la orientación y recursos para incluirlo, además de que el alumno tiene que ser copartícipe de este proceso. Igualmente se requiere que los docentes se formen en estrategias para la inclusión, que privilegien el trabajo colaborativo con sus colegas y, sobre todo, que tengan la disposición de acercarse y escuchar al educando.

Una segunda cuestión sobre la identificación es el diagnóstico, tema que algunos de los entrevistados consideraron fundamental para la planeación didáctica; no obstante, como hemos señalado, esto incrementa el riesgo de acotar la mirada solo en las deficiencias de los estudiantes. Para evitarlo es indispensable considerar que en la configuración de la discapacidad intervienen las barreras del entorno, de manera que más que preguntarse sobre lo que el alumno carece cabría cuestionarse sobre los obstáculos curriculares, físicos, pedagógicos y actitudinales que limitan sus aprendizajes y participación en la dinámica institucional.

En relación con el dilema de ubicación encontramos dos formulaciones, una referida a la factibilidad de que las personas con discapacidad cursen la educación superior y la otra vinculada con la carrera que deben estudiar. En ellas entran en juego, por un lado, los conocimientos y habilidades, en una palabra, el mérito de estos educandos para ingresar, mantenerse y obtener resultados equivalentes a los de sus compañeros, y por otro la capacidad que tiene la institución para atenderlos. Paralelamente está el asunto de si la discapacidad debería ser un elemento a considerar para la elección de la carrera y cuáles serían las probabilidades de que al terminarla pudieran encontrar un empleo acorde. En este caso conviene recordar que la educación es un derecho que tienen todas las personas, independientemente de su condición de vida o situación social, de manera que, si el estudiante cumplió con los requisitos institucionales, la discapacidad no tendría por qué ser un impedimento. Lo mismo podría aplicarse para el tema del mercado laboral, ya que, si bien se ha señalado que estas personas enfrentan más dificultades para ingresar a él, los alumnos sin discapacidad tampoco están exentos de ellas, y sin embargo este no es un factor que les impida estudiar la carrera que ellos eligen.

El análisis de estos dilemas nos permitió identificar algunos riesgos adicionales, entre ellos el refuerzo del estigma, sustentado en la idea de que estas personas nunca serán autónomas y por lo tanto abrirles un espacio en la universidad no garantiza que aprenderán o que lograrán concluirla; incluso que eventualmente pueden representar una presión más para el trabajo de los profesores y de la propia institución. Otro riesgo es confundir los apoyos y ajustes con prácticas paternalistas que limitan los aprendizajes y el desarrollo individual del estudiante; el punto de partida es el mismo, los prejuicios sobre la discapacidad y la manera en que incide en la autonomía de la persona. 
Uno de los hallazgos más significativos es que si bien los entrevistados no siempre reconocen a la inclusión como un proceso dilemático, sus inquietudes y dudas sobre el tema son recursivas. En ellas intervienen, entre otras cosas, el campo disciplinario del profesor, su historia profesional, su formación docente y el sentido que le da a su práctica. Esto nos lleva a afirmar que en las prácticas educativas y en la dimensión ética de su enseñanza la inclusión es un asunto relacional en el que los valores y los principios entran en juego, de manera que es importante fortalecer el perfil ético del profesor para que pueda identificar dichos dilemas y contar con elementos para afrontarlos.

En suma, los dilemas nos permitieron acercarnos a las elaboraciones que los profesores hacen con respecto a la discapacidad y a la inclusión, así como a las limitaciones que ellos observan tanto en el trabajo profesional como en las condiciones institucionales. En ese sentido es importante insistir en que la inclusión es un proceso que requiere la colaboración de los diferentes actores educativos, profesores, directivos, estudiantes con o sin discapacidad y personal de apoyo; los docentes son un agente central porque tienen el cometido de concretar los contenidos curriculares, con una población estudiantil cada vez más diversa. Para ello necesitan formarse, pero también contar con los recursos institucionales que les permitan construir prácticas y contextos educativos incluyentes.

Finalmente cabe señalar que el estudio de los dilemas en la inclusión educativa de personas con discapacidad es una veta de estudio que convendría explorar en el nivel superior, ya que, como hemos dicho, las investigaciones al respecto se centran en la educación básica. La identificación de los dilemas puede dar luces sobre las condiciones institucionales que rodean el proceso de enseñanza y aprendizaje, las barreras que enfrentan los estudiantes para ser incluidos plenamente y las áreas que necesitan fortalecerse en la formación de profesores. A nivel institucional, el análisis de los dilemas puede contribuir al desarrollo de políticas y procesos más incluyentes, por ejemplo, a partir de los resultados de esta investigación la universidad conformó un equipo de trabajo para revisar los procedimientos de admisión, tutoría y seguimiento académico e implementar acciones concretas que permitan fortalecer la inclusión de los alumnos con discapacidad y ampliar sus oportunidades de aprendizaje.

\section{REFERENCIAS}

Affonso, M., Collares, C., y Untoiglich, G. (2013). La máquina medicalizadora y patologizadora en la infancia. En M. Affonso, C. Collares, J. Geraldi, M. Terzaghi, C. B. Angelucci y R. Nunes, En la infancia los diagnósticos se escriben con lápiz. (pp. 25-44). Buenos Aires: Noveduc.

Ainlay, S., Becker, G., y Coleman, L. (1986). The dilemma of difference: A multidisciplinary view of stigma. Nueva York: Plenum.
Amaro, M., Méndez, J., y Mendoza, F. (2014). Un estudio de las características profesionales del docente universitario para atender a la diversidad. Revista Latinoamericana de Educación Inclusiva, 8(2), 199-216. Recuperado de http:// www.rinace.net/Rlei/numeros/vol8-num2/ art10.pdf. 
Artiles, A. (1998). The dilemma of difference: Enriching the disproportionality discourse with theory and context. The Journal of Special Education, 32(1), 3236. Recuperado de https://journals.sagepub.com/ doi/10.1177/002246699803200105.

Artiles, A. (2003). Special education's changing identity: Paradoxes and dilemmas in views of culture and space. Harvard Educational Review, 73(2), 164202. Recuperado de https://doi.org/10.17763/ haer.73.2.j78t573x377j7106.

Banks, S., y Williams, R. (2005). Accounting for ethical difficulties in social welfare: Issues, problems and dilemmas. British Journal of Social Work, 35, 1005-1022. Recuperado de https://www.researchgate.net/publication/31025922_.

Borland, J., y James, S. (1999). The learning experience of students with disabilities in higher education. A case study of a UK University. Disability \& Society, 14(1), 85-101. Recuperado de https://www.tandfonline.com/doi/abs/10.1080/09687599926398? journalCode $=$ cdso20.

Booth, T., y Ainscow, M. (2002). Index for inclusion. Developing learning and participation in schools. Bristol: CSIE.

Brunner, J. (2002). Educación superior: desafíos y tareas. Realidad y reflexión, (4), 61-81.

Brunner, J., y Flisfisch, A. (2014). Los intelectuales y las instituciones de la cultura. Santiago de Chile: Universidad Diego Portales.

Conapred [Consejo Nacional para Prevenir la Discriminación] (2010). Encuesta Nacional sobre Discriminación en México. México: Conapred. Recuperado de http://www.conapred.org.mx/userfiles/files/ Enadis-2010-RG-Accss-002.pdf.

Dyson, A., y Millward, A. (2000). Schools and special needs. Issues of innovation and inclusion. Londres: Sage.

Dolgoff, R., Harrington, D., y Loewemberg, F. (2012). Ethical decisions for social practice. Belmont: Books Cole.

Dubet, F. (2004). La escuela de las oportunidades. ¿Qué es una escuela justa? España: Gedisa.

Echeita, G. (2010). Alejandra L. S. o el dilema de la inclusión educativa en España. Murcia: Consejería de Educación, Formación y Empleo. http://diversidad.murciaeduca. es/tecnoneet/2010/docs/gecheita.pdf.

Etxeberria, X. (2005). Temas básicos de ética. España: Desclée.
Fuller, M., Bradley, A. y Healey, M. (2004). Incorporating disabled students within an inclusive higher education environment. Disability \& Society, 19(5), 455-468. Recuperado de https://www.tandfonline. com/doi/abs/10.1080/0968759042000235307.

Gairín, J., y Suárez, C. I. (2016). Inclusión y grupos en situación de vulnerabilidad: orientaciones para repensar el rol de las universidades. Sinéctica. Revista Electrónica de Educación, (46), 1-15. Recuperado de https:// www.redalyc.org/jatsRepo/ 998/99843455009/ $\mathrm{html} /$ index.html.

Getzel, E. (2008). Addressing the persistence and retention of students with disabilities in higher education: Incorporating key strategies and supports on campus. Exceptionality: A Special Education Journal, 16(4), 207-219. Recuperado de https:/ / www.researchgate. net/publication/240519376.

Goffman, E. (1970). Estigma. Argentina: Amorrortu.

INEGI [nstituto Nacional de Estadística y Geografía] (2013). Las personas con discapacidad en México, una visión al 2010. México: INEGI. Recuperado de: https://www.inegi.org.mx/app/biblioteca/ficha. html?upc $=702825051785$.

Juarros, M. F. (2006). ¿Educación superior como derecho o como privilegio? Las políticas de admisión a la universidad en el contexto de los países de la región. Andamios, 3(5), 69-90. Recuperado de https:// andamios.uacm.edu.mx/index. php/andamios/ article/view/342.

Kvale, S. (2011). Las entrevistas en investigación cualitativa. Madrid: Morata.

Latapí, P. (1993). Reflexiones sobre la justicia en la educación. Revista Latinoamericana de Estudios Educativos, 23(2), 9-41.

Miles, M., Huberman, M., y Saldaña, J. (2014). Qualitative data analysis: A methods sourcebook and the coding manual for qualitative researchers. Thousand Oaks: Sage.

Minow, M. (1990). Making all the difference. Ithaca: Cornell University Press.

Moola, F. (2015). The road to the ivory tower: The learning experiences of students with disabilities at the University of Manitoba. Qualitative Research in Education, 4(1), 45-70. Recuperado de https:/ / dialnet.unirioja.es/servlet/articulo?codigo $=5007462$. 
Norwich, B. (2008a). Dilemmas of difference, inclusion and disability. International perspectives and future directions. Londres: Routlege.

Norwich, B. (2008b). Dilemmas of difference, inclusion and disability: International perspective placement. European Journal of Special Needs Education, 23(4), 287-304. Recuperado de http://dx.doi. org/10.1080/08856250802387166.

Norwich, B. (2010). Dilemmas of difference, curriculum and disability: International perspectives. Comparative Education, 46(2), 113-135, Recuperado de https://www.tandfonline.com/doi/ abs/10.1080/03050061003775330.

Norwich, B. (2013). Addressing tensions and dilemmas in inclusive education: Living with uncertainty. Reino Unido: Routledge.

Nussbaum, M. (2007). Fronteras de la justicia. España: Paidós Ibérica.

OECD [Organización para la Cooperación y el Desarrollo Económicos] (2003). Disability in higher education. París: OECD.
Rodríguez, R. (1999). Expansión del sistema educativo superior en México 1970-1995. En M. Fresán, Tres décadas de políticas del Estado en la educación superior (pp. 167-205). México: ANUIES.

San Martín, C. (2011). ¿Dónde y cuándo proporcionar apoyos pedagógicos para facilitar los procesos de inclusión? Revista Latinoamericana de Educación Inclusiva, 5(2), 171-188. Recuperado de http:/ / repositoriocdpd. net:8080/handle/123456789/1914.

Stake, R. (1999). Investigación con estudio de casos. Madrid: Morata.

Terigi, F. (2009). Capítulo 1. La inclusión educativa: viejas deudas y nuevos desafíos. En F. Terigi, R. Perazza y D. Vaillant (coords.), Segmentación urbana y educación en América Latina (pp. 21-28). Madrid: Fundación Iberoamericana para la Educación la Ciencia y la Cultura.

UNESCO [Organización de las Naciones Unidas para la Educación, la Ciencia y la Cultura] (2009). Directrices sobre politicas de inclusión en la educación. UNESCO.

Valles, M. (1999). Técnicas cualitativas de investigación social. Reflexión metodológica y práctica social. Madrid: Síntesis.

Cómo citar este artículo:

Iturbide Fernández, P., y Pérez-Castro, J. (2020). Dilemas del profesorado en la inclusión educativa de estudiantes universitarios con discapacidad. IE Revista de Investigación Educativa de la REDIECH, 11, e1007. doi: https://doi. org/10.33010/ie_rie_rediech.v11i0.1007. 